\title{
PICTURE-BASED VIRTUAL TOURING
}

\author{
Weidong Geng, Yunhe Pan, Ming Li, Jian YANG \\ Zhejiang University, P R China
}

\begin{abstract}
This paper presents an image-based approach for virtual touring which use panoramas and picture-based animation to compose a virtual environment. Two typical touring behaviors, looking around and moving in one direction, are mimicked by players based on realtime image processing engine. This approach has been implemented in VRTOURISM. In addition to panoramic views, the system includes picture-based animation for moving forward to the next scene nodes. The paper describes the architecture, the authoring tools, and the interactive players of the VR system.
\end{abstract}

\section{Introduction}

Virtual touring is virtual navigation of landscapes that exists in the real world. One important application of virtual touring is in the promotion of tourism and presentation of landscapes. If we could create a virtual simulation of the landscape, and the user could interactively select touring paths and viewpoints, it would promote his/her desire to visit the real location, and would thus attract more tourists. These systems could also be distributed overseas by CDROM or placed on websites. It is more attractive than the traditional touring publicity using only video or still pictures.

Touring occurs primarily in two ways: looking around and moving in one direction. Looking around is referred to look the surrounding environment at one fixed location. This behavior is very similar to the camera panning. Moving in one direction resembles camera zooming within a scene. Walking along a specified touring path is a typical example.

We planned a virtual touring system to meet the following objectives. First, the above two typical touring behaviors should be mimicked simultaneously, and must be integrated seamlessly. Second, the system should play at interactive speed on most personal computers available today without relying on hardware acceleration, special input, or output devices such as data gloves or head-mounted displays We do not, however, prelude their use. Third, the system should focus on real scenes whose display speed should be independent of the rendering quality and scene complexity

Traditionally, virtual reality systems use 3D computer graphics to model and render virtual environments in real time [4,5]. However, real-world scenes (especially for natural landscapes) which contain enormously rich details are often difficult to model and render with a computer. This paper presents an approach that uses panoramic images integrated with a branching mechanism to compose a virtual environment. The panoramic images and branching pictures are digitally warped on-the-fly to simulate panning and zooming of the camera. 


\section{Related Work}

A key component in most virtual reality systems is the ability to perform a walkthrough of a virtual environment from different positions and orientations. The walkthrough requires the synthesis of the virtual environment and simulation of a virtual camera moving in the environment with up to six degrees of freedom. The synthesis and navigation are usually accomplished with one of the following three methods: 3D modeling and rendering, branch movies, and image-based rendering [1].

\section{$2.13 D$ MODELING AND RENDERING APPROACH}

A virtual environment is synthesized as a collection of 3D geometric entities. The geometric entities are rendered in real time, often with the help of special-purpose $3 \mathrm{D}$ rendering engine, to provide an interactive walkthrough experience [17-19]. This approach has three main problems. First, creating the geometric entities is a laborious manual process. Second, because the walkthrough needs to be performed in real time, the rendering engine usually places a limit on scene complexity and rendering quality. Third, the need for a special rendering engine has limited the availability of virtual reality for most people since the necessary hardware is not widely available. Despite the rapid advance of computer graphics software and hardware in the past, most virtual reality systems still face the above problems. The 3D modeling process will continue to be a very human-intensive operation.

\subsection{BRANCHING MOVIES APPROACH}

Multiple movie segments depicting spatial navigation paths are joined at selected branch points. The user is allowed to move to a different path only at these branching points. This approach usually uses photography or computer rendering to create the movies. A computer driven analog or digital video player is used. An early example of this approach is the movie-map [3], in which the streets of the city of Aspen were filmed at 10-foot intervals. At playback time, two videodisc players were used to retrieve corresponding views to simulate the effects of walking on the streets.

An obvious problem with the branching movie approach is its limited navigability and interaction. It requires a large amount of storage space for the movies corresponding to all possible choices. It solves the problems mentioned in the 3D approach. It does not require 3D modeling and rendering for existing scenes. This approach allows rendering to be performed at the highest quality with the greatest complexity without affecting the playback performance. It can also use inexpensive and common video devices for the playback.

\subsection{IMAGE-BASED RENDERING APPROACH}

Quick Time VR is the first commercial product using the image-based navigation method [1]. It is an image-based rendering system for virtual environment navigation based on the above objectives. The system uses real-time image processing to generate 3D perspective viewing effects. This approach is very similar to the movie-based approach and shares the same advantages. It differs in that the movies are replaced with "orientation-independent" images and the movie player is replaced with real-time image processing. The images currently used are orientation-independent because each of the images contains all the information needed to look around in the 360 degrees. A number of these images are connected to form a walkthrough sequence. 


\section{Simulation of Typical Touring Behaviors}

The image-based approach presented in this paper addresses the simulation of virtual camera motions in photographic spaces. Free motion of the camera in a space, termed "camera movement", requires the change of both the viewpoint and the view direction. A camera moving freely within a scene involves the change of viewpoint and view direction. The view direction change can be accomplished with the use of an environment map. The viewpoint change is more difficult to achieve. A simple solution to viewpoint change is to constrain the camera's movement to only particular locations where environment maps are available. For a linear camera movement such as walking down a hallway, environment maps can be created for points along the path at some small intervals. Changing the camera's field-of-view, termed "camera zooming", can be accomplished through multiple-resolution image zooming. Changing the camera's field of view is equivalent to zooming in and out in the image space.

The two typical touring behaviors, looking around and moving in one direction, are realized by two players in our virtual touring system VRTOURISM. The panoramic player is used to mimic the "looking around" behavior. It is similar to that of QUICKTIME-VR in that the walking-through player, based on picture-based animation [2], is used to mimic the "moving in one direction" behavior.

\subsection{LOOKING AROUND}

The mimicking of looking around is based on panoramic images. It is shown in Figure 1. The window in panorama is used to simulate the movement of the tourist's eyes. The size of window is dependent on the view field, and is manually specified by the user. The scene is composed of a set of panoramic nodes organized in branching mechanism. The principle to connect the panoramic nodes is that there exists at least one image in the nodes to be linked to the next image which is captured in the same (or nearly same) viewing direction. These images are called the "hotspot images" in our system, and are utilized by the simulation of moving in one direction.

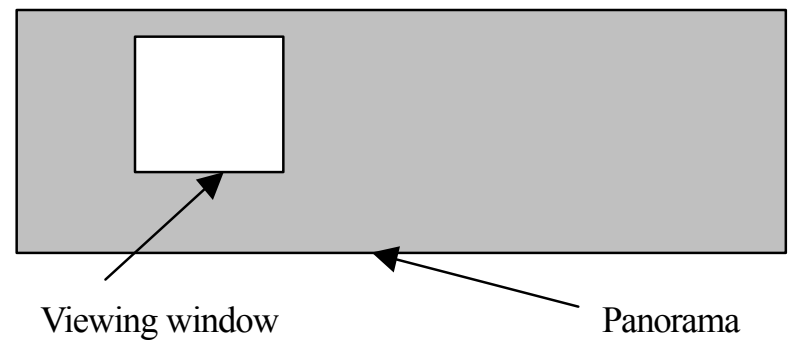

Figure 1 Simulation of eye movement

Panoramic movies permit a user to pan and move in a space interactively. The panoramic player allows the user to perform continuous panning in the horizontal directions. Because the panoramic image has less than 180-degrees vertical field-of-view, the player does not permit looking all the way up to view. Rotating about the viewing direction is not currently supported. The performance of the player varies depending on many factors such as the platform, the panning mode, and the window sizes. The panoramic player also allows the user to control the view orientation and display a perspectively correct view by warping the panoramic image. An example of touring of the West Lake is shown in Figure 2. 


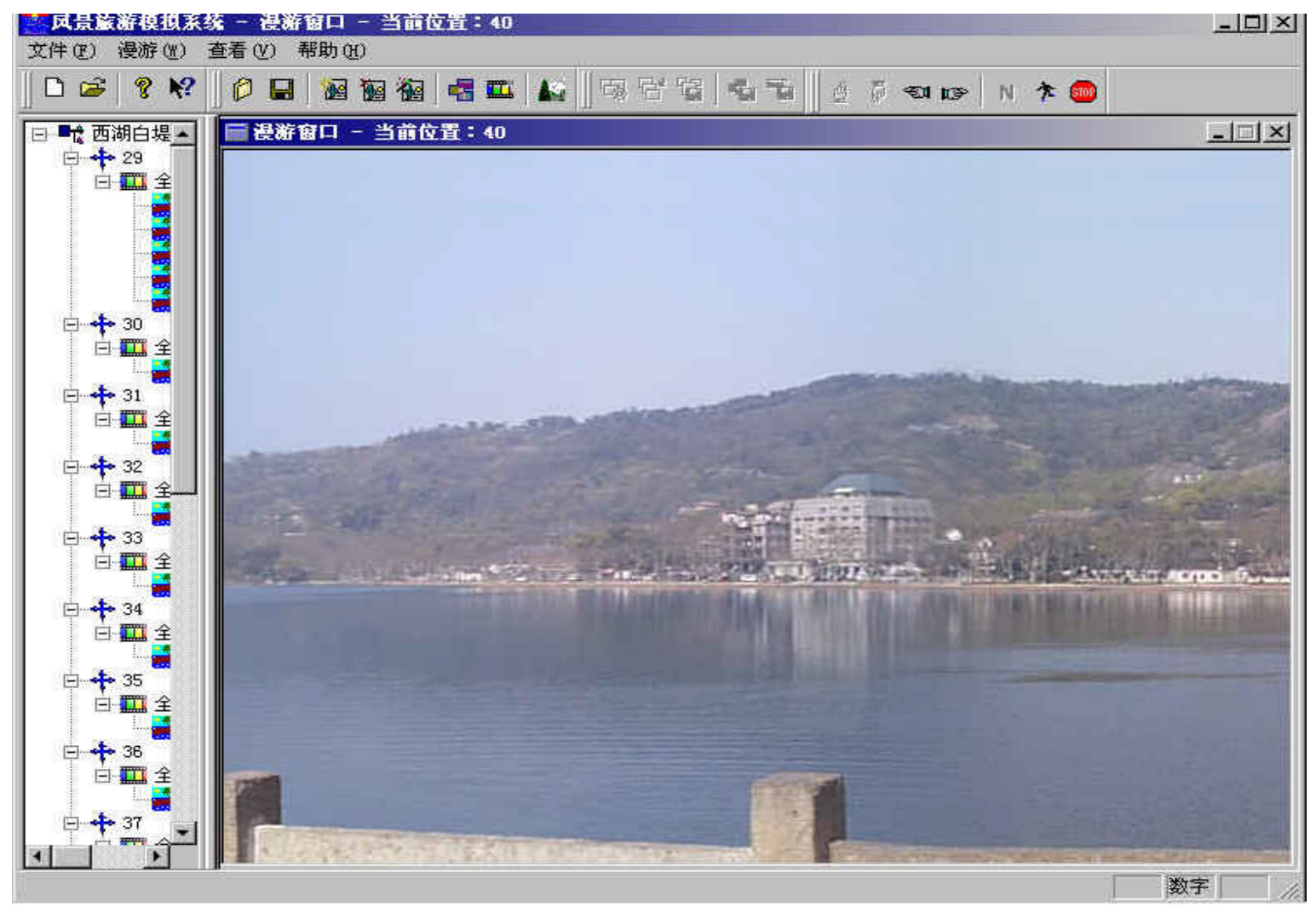

Figure 2 The interface of panoramic player in VRTOURISM

\subsection{MOVING IN ONE DIRECTION}

In QUICKTIME-VR, moving in space is accomplished by jumping to points where panoramic images are attached. In order to preserve continuity of motion, the view direction needs to be maintained when jumping to an adjacent location. The panoramas are linked together by matching their orientation manually in the authoring stage.

In VRTOURISM, picture-based animation approach [2] is used to mimic the moving in one direction. The local 3D model (shown in Figure 3) is first recovered from the epipolar point and the internal rectangle specified by the user in the authoring stage. The animation sequence is generated from the local model of neighboring hot images. Perceived depth during the motion is corrected by the local 3D model. Additional models could also be added to the local model as an improvement of the spidery model in [2]. The local 3D model consists of a set of 3D boxes, and the image is assumed as the texture mapped on it. In Figure 3, the floor may be considered as the road for touring. A and B are the additional models to approximate the near landscape along the road. In order to achieve a smoother visual perception of moving, the empirical restriction on the neighboring hotspot images is that along the walking direction the area of sub-regions, which are the same in content, is greater than half of the area of the corresponding hotspot images. 


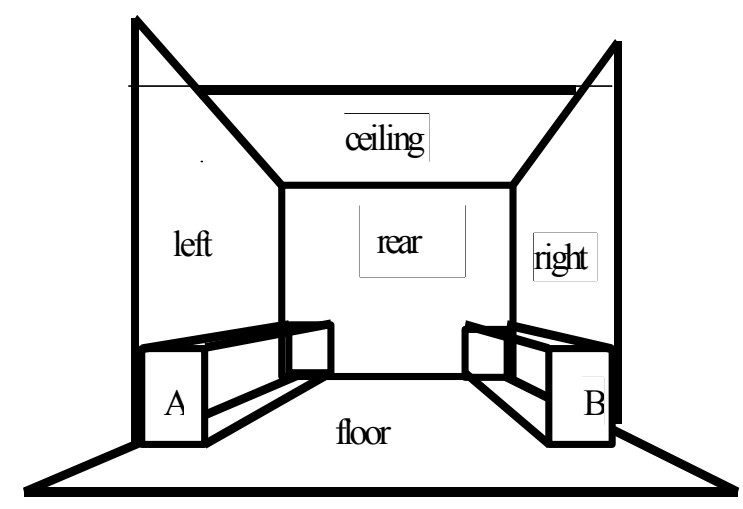

Figure 3 An improvement over the spidery model described in ref [2]. $A$ and $B$ are additional models of near landscape along the floor

\section{The Authoring Environment}

The authoring environment includes tools to make panoramic movies, specify the correspondence of preand-aft adjoining pictures, and generate the local model to be utilized in simulation of continuously moving forwards. The tools in the authoring environment are shown in Figure 4.

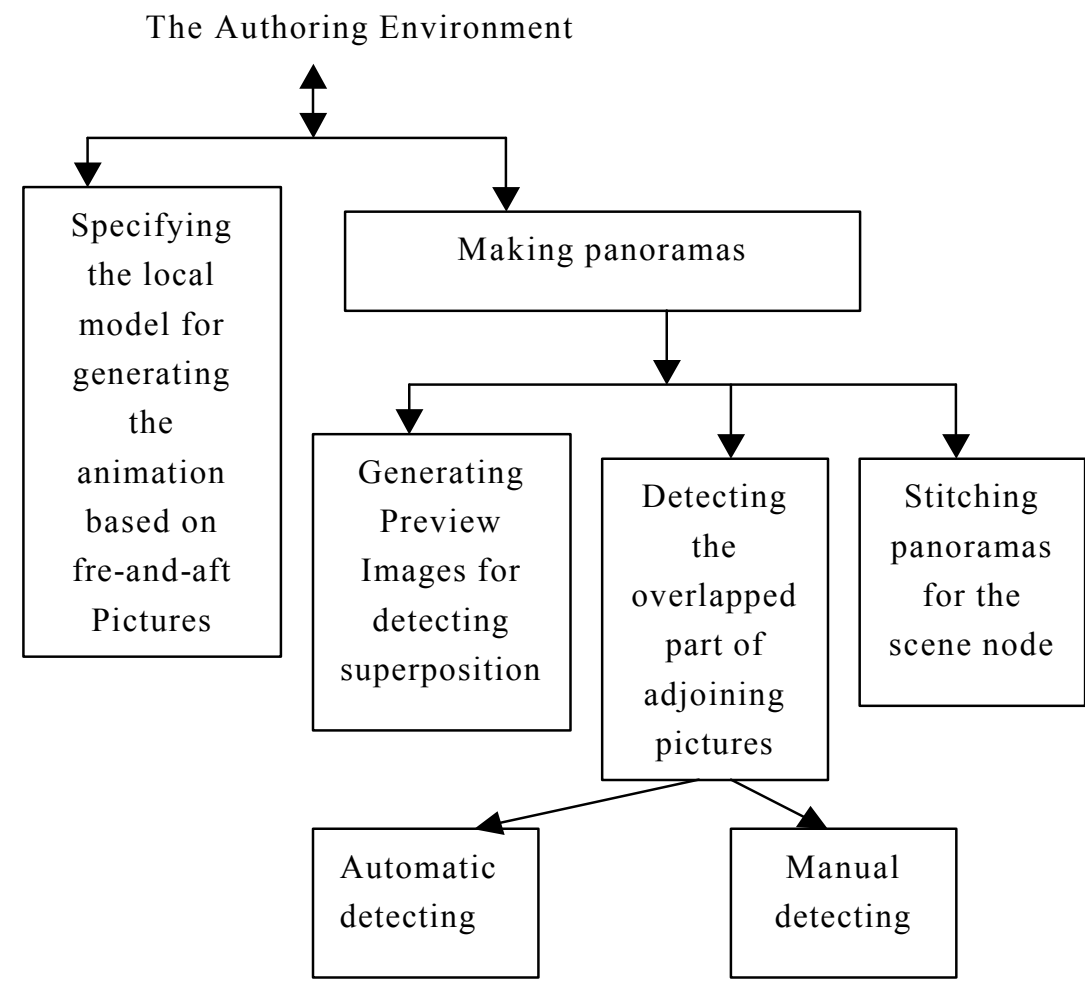

Figure 4 Tools for authoring in VRTOURISM

The key to panorama-making is to find overlapping parts of the adjoining pictures. In VRTOURISM, that is performed on downsized pictures. Locating of the superposition is realized in two modes: manual and automatic. Manual locating is performed by mouse drag, and the overlap parts are rendered transparently to enable the user to align the position easily. 
The software searches the region of superposition automatically with some parameters specified by the user. The principle of the auto-stitching is that the difference in intensity of the images' superposition should be zero. In fact, the difference is near zero but cannot be exactly zero because of noise. Since the images are taken horizontally in our system, we only need to consider the shift in 2D directions - $\mathrm{X}$ and $\mathrm{Y}$. On the assumption that the area of the overlapped portion is $\mathrm{S}$, the "energy" $E$ is

$$
E=\frac{\sum_{i}\left|I_{2 i}-I_{1 i}\right|}{S}
$$

Its numerical optimization is completed by the block-based search algorithm in computer vision [16]. In order to improve the robustness and find the superposition quickly and correctly, the starting point and end point for searching: (xStart, yStart) and (xEnd, yEnd), are specified initially. The user is also allowed to specify the step(nStep) and the number (nCount) of the search. The next search step modifies $\log _{2}$ (nStep) until it reaches one, and the numerical optimization process then terminates.

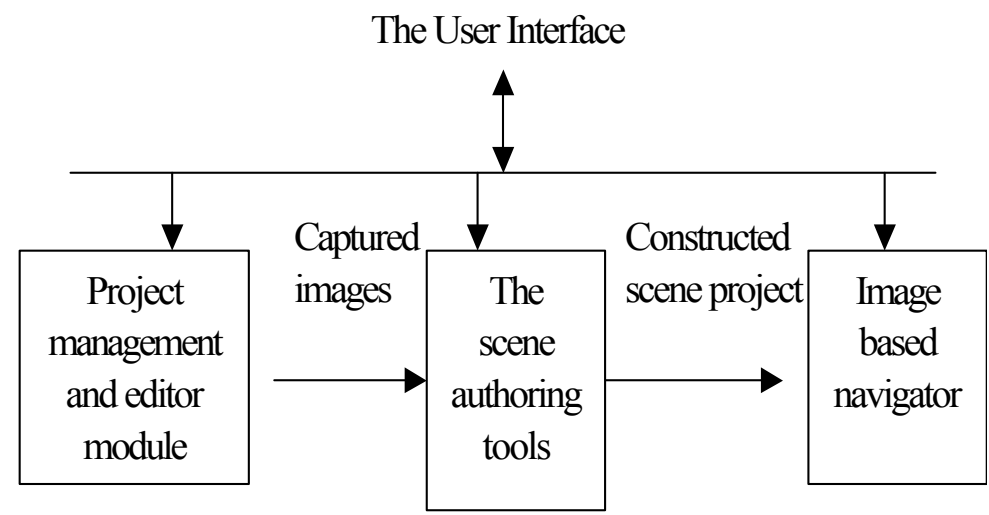

Figure 6 The architecture of VRTOURISM

A recommended approach for locating is to adjust the superposition manually on the basis of automatic search. When the locating is finished, the input pictures are stitched to generate one panorama based on the overlapped parts. The panorama-making process is shown in Figure 5.
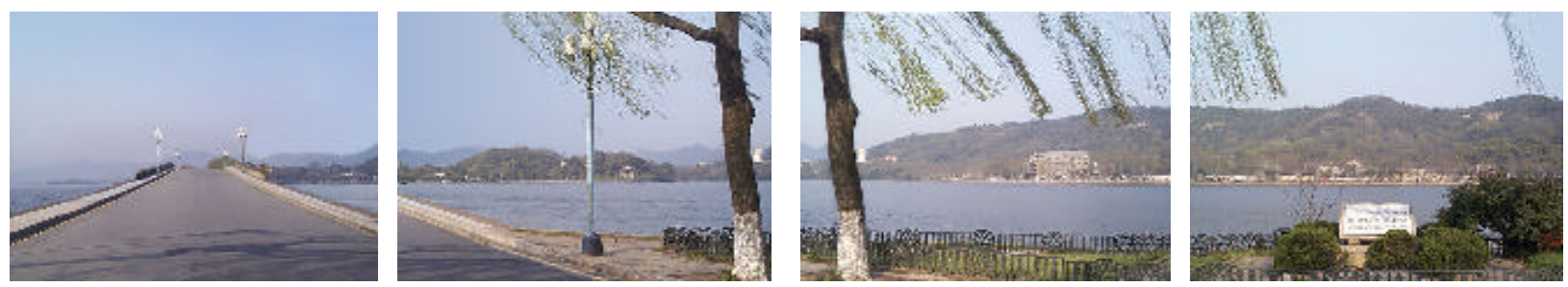

Figure 5 (a) Captured images 


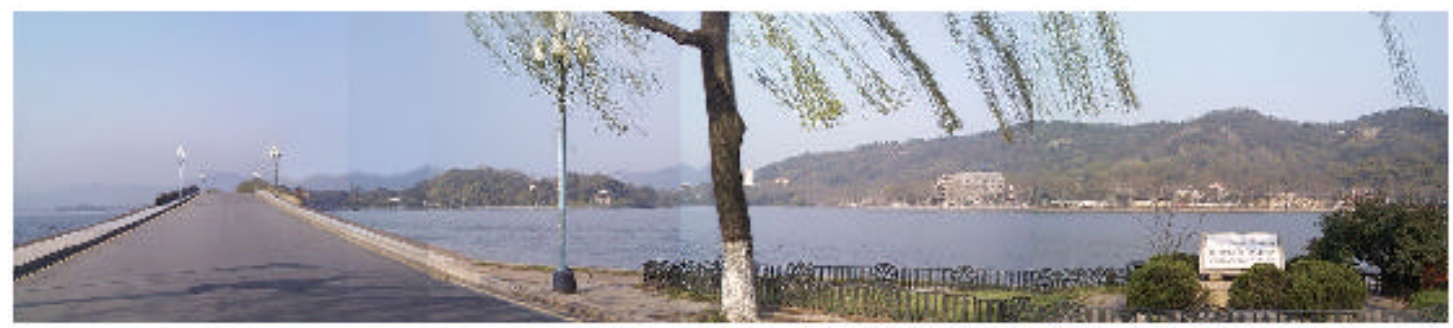

Figure 5 (b) The overlapped parts detected are rendered transparently.

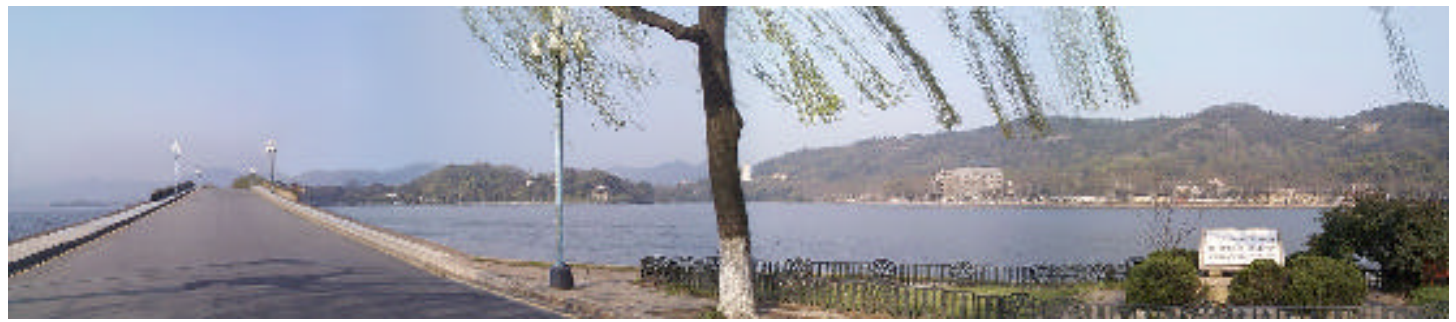

Figure 5 (c) The stitched panoramas

Figure 5 The panorama-making process

The purpose of stitching is to create a seamless panoramic image from a set of overlapped pictures. The pictures are taken with a camera as it rotates about its vertical axis in one direction only. The stitcher uses a correlation-based image registration algorithm to match and blend adjacent pictures. The adjacent pictures need to have some overlap for the stitcher to work properly. The amount of overlap may vary depending on the image features in the overlapping regions.

To eliminate the color discontinuities of overlapped parts between the two adjacent images, a weight-based feathering method is utilized during stitching. The weight of the pixels in each image proportionally to their distance to the center point of the image is given by

$$
d \text { Weight }=\cos \left(\frac{\pi}{2} \cdot\left|\frac{x-X_{0} / 2}{X_{0} / 2}\right|\right) \cdot \cos \left(\frac{\pi}{2} \cdot\left|\frac{y-Y_{0} / 2}{Y_{0} / 2}\right|\right)
$$

where $d$ Weight is the corresponding weight of the pixel $(x, y)$ and $(X o, Y o)$ is the image center. The blended color of the overlapped part is calculated according to the equation.

$$
I_{r(g, b)}=I_{r(g, b) 1} \cdot d \text { Weight }_{1}+I_{r(g, b) 2} \cdot d \text { Weight }_{2}
$$

If more than one panoramic node is needed, the panoramas are linked together by manually registering their viewing directions, and a hot-spot image is specified by marking regions of panorama with rectangles. The permitted touring paths in this scene are extracted from the links. The user should specify the correspondent relation of contents in the fore-and-aft picture by defining the control rectangle and specifying the epipolar point as shown in Figure 3. The local model to correct 
the depth of moving to the next panoramic node is recovered from it. The corresponding relationships of content are utilized in interpolation of pictures. The nodes should be selected to maintain visual consistency when moving from one to another. The distance between two adjacent nodes is related to the size of hot image and the distance to the nearby objects. In hot spot regions two typical touring behaviors are both allowed.

\section{The Virtual Touring}

\section{System: VRTOURISM}

The virtual touring system, VRTOURISM, includes an interactive environment which uses a software-based realtime image processing engine for navigating in space and an authoring environment for creating VR movies. The interactive environment is implemented using $V C^{++} 6.0$ and Open Inventor. The interactive environment comprises two types of players. The panoramic player is used to simulate the "Looking Around" behavior. It allows the user to pan and navigate within a scene. It also allows the user to interact in the navigation process. The walking-through player is used to mimic the "moving in one direction" behavior. It allows the user to go to the next scene node "smoothly". It includes two components: one is used to correct the perspective depths of the neighboring hotspot images. Only in the hotspot image can the user be allowed to go to the next scene node. The other is to perform a picture-based-animation operation to simulate the smooth change of image contents between the neighboring hotspot images. The authoring environment consists of a suit of tools to perform panoramic image stitching, marking, linking, and specifying the position relations of neighboring images, thereby establishing the correspondence between the neighboring hotspot images.

The architecture of VRTOURISM is shown in Figure 6. The project management and editor is responsible for creating a new project, organizing captured images, and specifying their correlations, i.e. left, right, front, and rear in the project. The scene authoring is used to construct the scene model based on the captured images in the project. The navigator allows the user to choose the touring modes: auto-touring or interactive touring. During touring the users can change the touring path, direction, speed, and step according to his/her interests at any time. The components to implement function of navigator are shown in Figure 7. The details for navigating and authoring in VRTOURISM are shown in Figures 8 and 9.

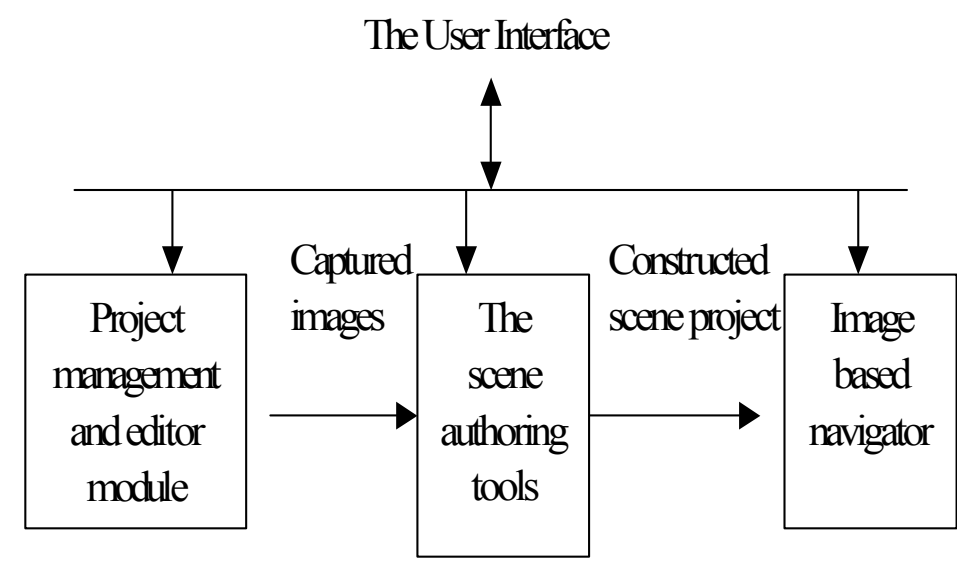

Figure 6 The architecture of VRTOURISM 
The Interactive $\mathrm{N}$ avigator

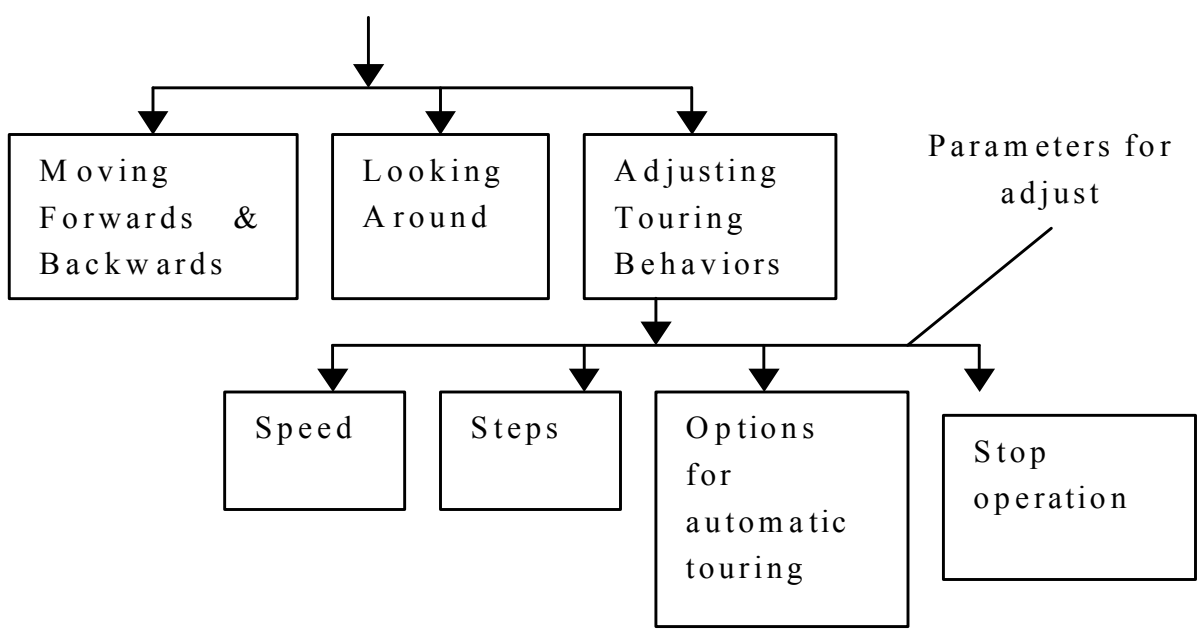

Figure 7 The functional components in the interactive navigator

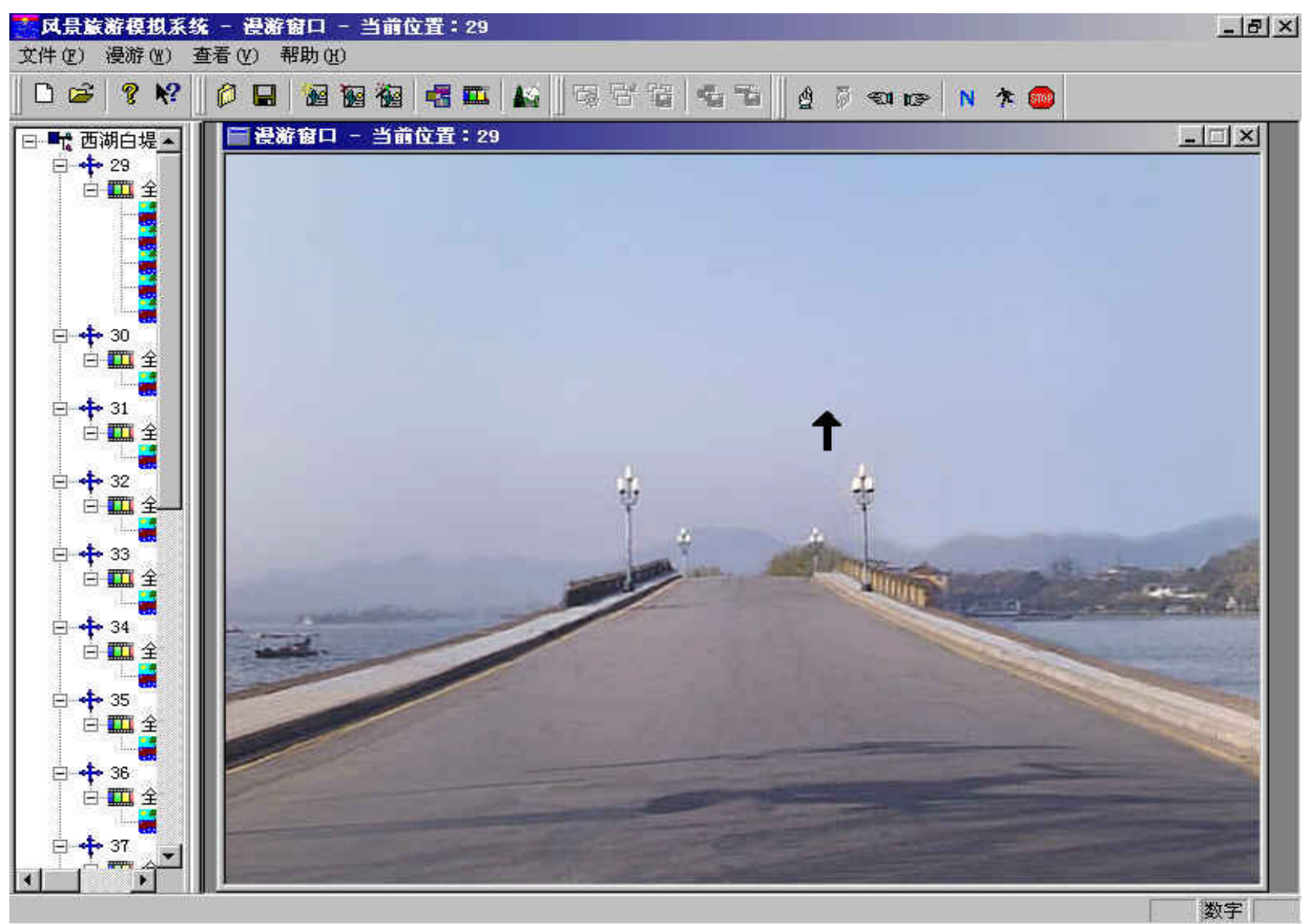

Figure 8 The navigation interface 


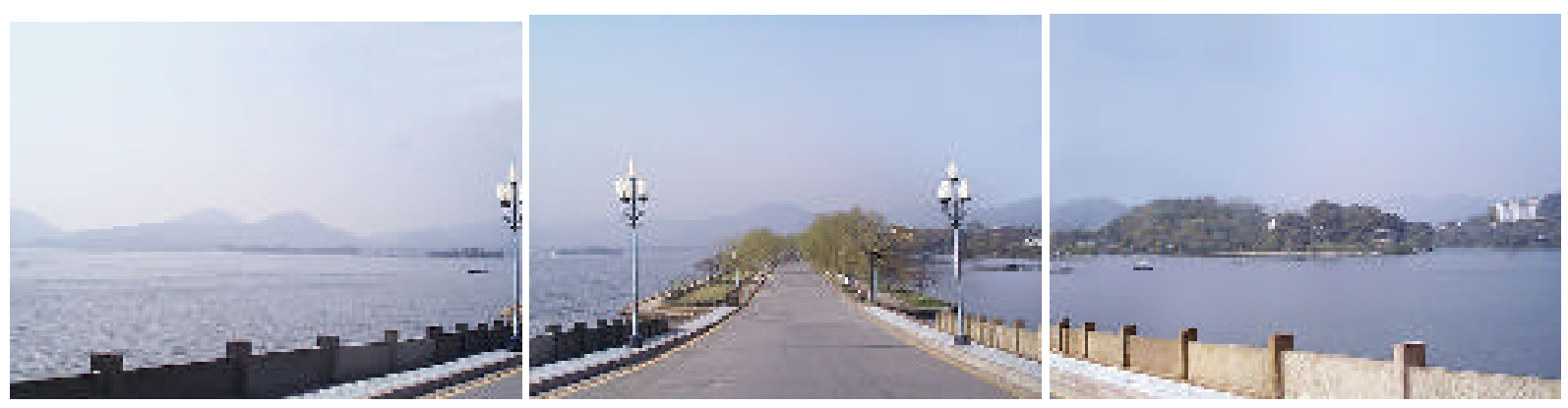

Figure 9 (a) The captured images

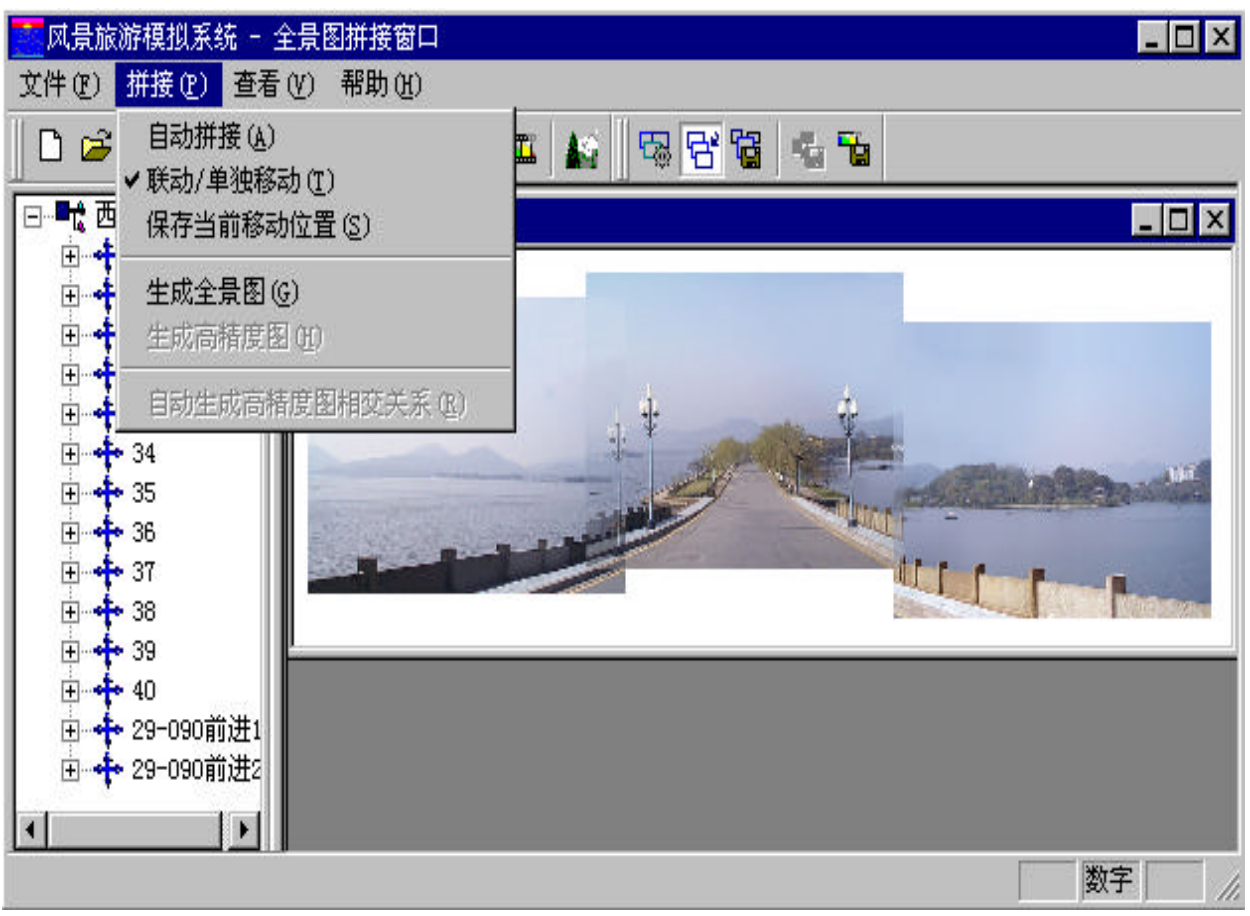

Figure $9(b)$ The interface for making the panorama

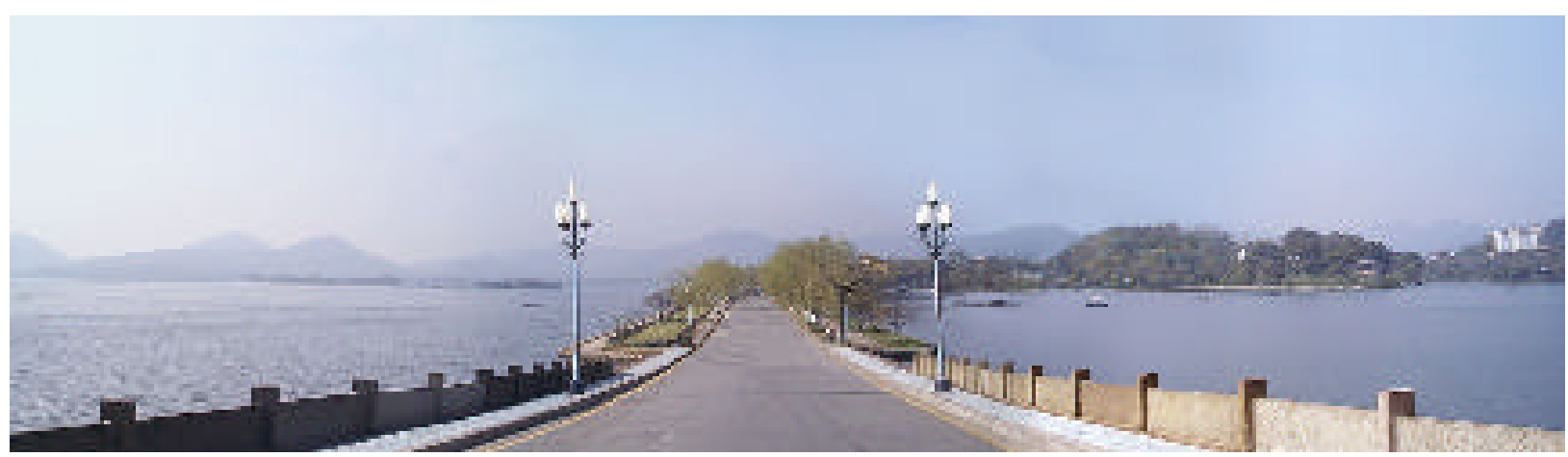

Figure 9 (c) The final panorama

Figure 9 Making the panorama in VRTOURISM 


\section{Discussion and Conclusion}

Two typical touring behaviors, looking around and moving in one direction, are supported in our virtual touring system VRTOURISM. Panoramas integrated with branching picture-based-animation are used to compose the scene. The related image processing techniques such as view morphing and view interpolation are incorporated in the touring navigator [6-15]. Looking around is based on panoramas. Moving in one direction is realized by the picture animation based on the improved spidery model. However, the touring path extracted in VRTOURISM are simplified to straight lines for picture-based animation. It has been applied to the virtual touring of The West Lake.

The chief limitations of this method are the requirements that the scene should be static and the movement be confined to particular scene nodes. Being able to move freely in a photographic scene is more difficult, although we can achieve a smoother visual effect than that of QUICKTIME-VR when moving to the next scene nodes.

Compared with virtual touring based on video, the role of the user is different, and fewer data are needed. In video based touring, the user is presented with video passively. In VRTOURISM, the user can actively select the touring path or wander in the scene at will. Compared with the touring in 3D graphic world, it is more suitable for the virtual touring of natural landscapes, for there is no laborious work of 3D modeling.

\section{Acknowledgement}

The work is partly supported by the Local Key Project of for Zhejiang Province. The author is grateful to the entire VRTOURISM team for their tremendous efforts on which this paper is based. Thanks for Dr. Pan Zhigeng's suggested changes of the paper.

\section{REFERENCE}

[1] Chen, S. E. "QuickTime VR-An Image-based Approach to Virtual Environment", Proc. SIGGRAPH'95, pp29-38

[2] Horry, Y, Aniyo, K and Arai, K "Tour Into the Picture: Using a Spidery Mesh Interface to Make Animation from a Single Image", Proc. SIGGRAPH'97, pp225-232

[3] Lippman, A "Movie Maps: An Application of the Optical Videodisc to Computer Graphics", SIGGRAPH'80, pp32-43

[4] X.H. Ma, Z.G. Pan, M.M. Zhang, et al. "The Automatic Generation of Multiple LoD and its Application", Progress in Natural Science, No.3, 1998

[5] Z.G. Pan, M.M. Zhang, W.T. Zheng et al. "Time-critical Computing in Virtual Environment"'. Proceedings of CAD/Graphics'95, Wuhan, China, Oct. 1995,1074-1079

[6] Chen, S E and Williams, L "View Interpolation for Image Synthesis", Proc. SIGGRAPH'93, pp279-288

[7] Devebec, P E, Taylor, C. A. and Malik, J "Modeling and Rendering Architecture from Photographs: A Hybrid Geometry and Image Based Approach”, Proc. SIGGRAPH'96, pp11-20

[8] Shade, J and Gortler, S "Creating Full View Panoramic Image Mosaics and Environment Maps”, Proc. SIGGRAPH'97, pp251-258

[9] Szeliski, R and Shum, H "Video Mosaics for Virtual Environment Maps", IEEE CG\&A, pp 2230, march, 1996 
[10]McMillan, L., and Bishop, G. Plenoptic modeling:An Image-based rendering system.. Proc. SIGGRAPH 95. pp. 39-46.

[11]Seitz, S.M., and Dyer, C.R. "View Morphing” Proc. SIGGRAPH 96 , pp.21-30.

[12]Beier,T.,and Neely, S. Feature-based image metamorphosis. Proc. SIGGRAPH 92. pp. 35-42.

[13]Tsai R.Y and Lenz R.K., "A new technique for fully autonomous and efficient 3D robotics hand/Eye calibration," IEEE Trans. on Robotics and Automation, Vol.5, No.3, June 1989, pp. 345-358.

[14]Saied Moezzi, Arun Katkere, Don Y.Kuramura, Ramesh Jain: Reality modeling and visualization from multiple video sequences. IEEE Computer Graphics and Applications. November 1996.

[15] Oliver Grau, A scene analysis system for the generation of 3-D models. Proceedingsof International Conference On Recent Advances In 3-D Digital Imaging and Modeling. pp 221228. 1997.

[16]Ma Songde, Computer Vision, The Science Press: Beijing, 1998

[17]D.Xu, Z.G. Pan, J.Y. Shi, " Image-based rendering techniques for VR", Journal of Image and Graphics, 3(12):1005-1010, 1998

[18]Z.G. Pan, J.Y. Shi , M.M. Zhang “ Distributed Graphics Support for Virtual Environment”, Computers \& Graphics, 20(2), 1996

[19]Zhigeng Pan, Jiaoying Shi, Qin Lu, " Virtual Reality Technology Development in China: An Overview", IJVR, 4(4), 1999

\section{BIOGRAPHIES}

Weidong Geng is an Associate Professor of the Department of Computer Science and Engineering, Zhejiang University. He received a BS degree from Nanjing University, an MS degree from National University of Defense Technology and a PHD degree from Zhejiang University. He has authored or co-authored nearly 30 papers. His current research interests include CAD/CG, AI, and multimedia.

\section{Contact information:}

Dr. Weidong Geng

Dept. Of Computer Science \& Engineering

Zhejiang University

Hangzhou, 310027, PR China

Phone:+86-571-7951248

Fax: + 86-571-7951799

E-mail: mailto:gengwd@cs.zju.edu.cn 
Yunhe Pan is a Professor of the Department of Computer Science and Engineering, Zhejiang University. Currently he is the President of Zhejiang University, the Director of Artificial Intelligence Institute of Zhejiang University. He received an MS degree from the Department of Computer Science and Engineering of Zhejiang University. Since the 1980s his work has concentrated on intelligent CAD, computer art, multimedia and VR. He has published more than 200 papers.

\section{Contact information:}

Prof. Yunhe Pan

President Office

Zhejiang University, Hangzhou, China

Phone: +86-571-7951109

Fax: $+86-571-7951358$

Email: mailto:Panyh@sun.zju.edu.cn

Ming $\mathbf{L i}$ is an MS candidate at the Intelligent CAD Lab of Zhejiang University. He received a BS degree from Zhejiang University in Computer Software. His current research interests include Image-based Modeling, Augmented Reality.

Jian Yang is an MS candidate at the Intelligent CAD Lab of Zhejiang University. He received a BS degree from Zhejiang University in Computer Software. His current research interests include Image-based Rendering, Image processing. 